\title{
Automatic greenhouse pest recognition based on multiple color space features
}

\author{
Zhankui Yang ${ }^{1,2,3}$, Wenyong $\mathrm{Li}^{2,3}$, Ming $\mathrm{Li}^{2,3}$, Xinting Yang ${ }^{2,3 *}$ \\ (1. College of Computer Science and Technology, Beijing University of Technology, Beijing 100124, China; \\ 2. National Engineering Research Center for Information Technology in Agriculture, Beijing 100089, China; \\ 3. National Engineering Laboratory for Quality and Safety Traceability Technology and Application of Agricultural Products, \\ Beijing 100089, China)
}

\begin{abstract}
Recognition and counting of greenhouse pests are important for monitoring and forecasting pest population dynamics. This study used image processing techniques to recognize and count whiteflies and thrips on a sticky trap located in a greenhouse environment. The digital images of sticky traps were collected using an image-acquisition system under different greenhouse conditions. If a single color space is used, it is difficult to segment the small pests correctly because of the detrimental effects of non-uniform illumination in complex scenarios. Therefore, a method that first segments object pests in two color spaces using the Prewitt operator in I component of the hue-saturation-intensity (HSI) color space and the Canny operator in the B component of the Lab color space was proposed. Then, the segmented results for the two-color spaces were summed and achieved $91.57 \%$ segmentation accuracy. Next, because different features of pests contribute differently to the classification of pest species, the study extracted multiple features (e.g., color and shape features) in different color spaces for each segmented pest region to improve the recognition performance. Twenty decision trees were used to form a strong ensemble learning classifier that used a majority voting mechanism and obtains $95.73 \%$ recognition accuracy. The proposed method is a feasible and effective way to process greenhouse pest images. The system accurately recognized and counted pests in sticky trap images captured under real greenhouse conditions.
\end{abstract}

Keywords: ensemble learning classifier, greenhouse sticky trap, automated pest recognition and counting, HSI and Lab color spaces, multiple color space features

DOI: $10.25165 /$ j.ijabe.20211402.5098

Citation: Yang Z K, Li W Y, Li M, Yang X T. Automatic greenhouse pest recognition based on multiple color space features. Int J Agric \& Biol Eng, 2021; 14(2): 188-195.

\section{Introduction}

Pest management is one of the main concerns for agricultural scientists and producers. Pests affect crop yield and quality and require substantial manpower and material resources for management. The automatic recognition and counting of pests is an essential step toward effectively managing pests and improving resource utilization efficiency ${ }^{[1]}$. Typically, greenhouse pest recognition and counting are conducted on conventional sticky traps $^{[2]}$. The small size of the pests and the visual complexity of the greenhouse sticky traps (such as illumination and impurities) reduce the efficiency and reliability of manual counting. With the development of agricultural information technology, an integrated pest management system was proposed to minimize crop damage, environmental pollution, and economic losses ${ }^{[3]}$. However, one of the prerequisites for integrated pest management is to accurately investigate pest species as well as population density. Thus, this paper introduces our automatic greenhouse pest recognition system.

The rapid development of computer vision technology has

Received date: 2019-04-22 Accepted date: 2019-12-25

Biographies: Zhankui Yang, $\mathrm{PhD}$, research interest: machine learning, Email: a437112007@163.com; Wenyong Li, PhD, research interest: agricultural informatization, Email: liwy@nercita.org.cn; Ming Li, PhD, research interest: agricultural informatization, Email: liming@nercita.org.cn.

*Corresponding author: Xinting Yang, $\mathrm{PhD}$, Professor, research interest: agricultural informatization. National Engineering Research Center for Information Technology in Agriculture, Beijing 100089, China. Tel: +8615010740057, Email: yangxt@nercita.org.cn. facilitated a new pest recognition approach. The use of an automated approach greatly increases efficiency and addresses problems such as a lack of agriculture experts and poor objectivity ${ }^{[4]}$. Though image processing methods are widely used for the automated recognition and counting of various objects in different fields, very few studies have been conducted on recognizing and counting pests on sticky traps. The related literature can be grouped along several dimensions such as image acquisition settings, segmentation, feature extraction, and classification algorithms. In terms of image sources, many previous methods have considered individual pest specimens ${ }^{[5-9]}$ or pest images captured under laboratory conditions ${ }^{[10-13]}$. Thus, when these existing methods are applied to pest images captured in real-world scenarios, they may yield low recognition accuracy. Some previous methods have been used to perform pest image acquisition in a real greenhouse environment using scanners or wireless digital cameras. From an image acquisition perspective, the light source in a scanner is consistent and the image quality is uniform $^{[1,14,15]}$. However, scanners are mainly used indoors, and using a scanner in the field is inconvenient. Pest image acquisition using a digital camera or a CCD camera is very common $^{[16-19]}$. Although these methods achieve high pest recognition accuracy, they are not specifically suitable for large-scale implementation and long-term pest monitoring because of the high computational cost for data processing. In addition, the cameras in these systems do not have data transmission capabilities, thereby hindering the installation of a complete monitoring system.

Previous studies have suggested analyzing and counting pests 
on only $20 \%$ of the trap surface rather than on the complete surface to provide an accurate estimation of overall pest density ${ }^{[20,21]}$. Existing methods use solid-color traps to avoid noise in the digital images caused by gridlines or other printed marks on the sticky traps ${ }^{[15,22,23]}$. In contrast, our proposed segmentation algorithm can deal with non-uniformly colored sticky traps. Cho et al. and Qiao et al. use empirical intensity thresholds to segment pests in the trap images, however, the empirical parameter requires manual adjustment if the method is used under different image acquisition conditions ${ }^{[1,22]}$. Solis-Sánchez et al. used the automatic clustering-based image threshold method proposed by Otsu (1979) for whitefly image segmentation from sticky trap images captured in the field, thereby reducing the empirical input parameters used in the insect identification algorithm ${ }^{[24]}$. Similarly, Xia et al. used a marker-controlled watershed segmentation. A comparative experiment was performed to verify that our proposed algorithm provides better segmentation results on the pest trap images than the watershed segmentation algorithm ${ }^{[15]}$.

Hand-crafted features, including the scale-invariant feature transform (SIFT) and histogram of gradients (HOG), have facilitated dramatic progress in many computer vision tasks such as object recognition and image matching. These features are considered to be a major milestone in computer vision and perform consistently. Solis-Sánchez et al. identified the objects detected in the segmentation process using SIFT as developed by Lowe ${ }^{[25,26]}$. Xiao et al. presented a pest classification and recognition scheme based on the SIFT and bag-of-words model ${ }^{[27]}$. Deng et al. generated saliency maps and regions of interest-based on the saliency-using-natural-statistics model, and then fed the invariant features to a support vector machine (SVM) for recognition ${ }^{[28]}$. These methods have achieved accurate results, but they rely on the extraction of SIFT features, which entails a significant computational burden and is not feasible for our online real-time identification system.

Currently, there is no unified method for image segmentation and recognition in greenhouse conditions. This study using color and shape features to identify the pests from regions detected in the segmentation process. A novel multi-decision tree ensemble learning method was adopted for classification, which constructs multiple classifications and regression trees (CART) by random resampling on the feature variables, and classifies the pests using multiple decision-tree voting. Our study makes three main contributions. First, a 4G network mobile or wireless network transmission solution was proposed to transmit images on a large scale and a set of energy storage devices were designed to run image acquisition devices with little manual intervention. Second, an accurate recognition and counting algorithm with a low computational cost for pests on sticky trap images captured in a greenhouse environment was developed. Third, the agricultural monitoring web platform was developed to monitor the function of the terminal device and view the recognition and counting results of greenhouse pests in real-time.

\section{Materials and methods}

\subsection{Samples and image acquisition}

All of the images are collected from a cucumber greenhouse at the National Experiment Station for Precision Agriculture in Beijing, Jinggangshan National Technology Park in Jiangxi, and the Science Park in Tianjin. Figure 1a shows the complete acquisition and transmission system using a T500 industrial mobile phone (Figure 1c), as deployed in the greenhouse environment.

The automated image acquisition system in Figure 1a consists of the following components from top to bottom: 1) a solar panel: the power supply for the mobile phone consists of a solar panel combined with a storage battery; 2) a sticky trap: the trapping section is $20 \mathrm{~cm} \times 25 \mathrm{~cm}$ and is attached by a bracket in the middle of the monitoring device; 3 ) a camera box: the image acquisition device is directly in front of the sticky trap, and the image is captured by a T500 three-proof mobile phone and transferred to our monitoring service platform by setting the photo time interval in the $4 \mathrm{G}$ or wireless network environment; and 4) a battery box: a $20000 \mathrm{~mA} \cdot \mathrm{h}$ battery combined with the solar panel facilitates quick electricity storage. Figure 2 shows a schematic diagram of the entire system as deployed in the greenhouse environment.

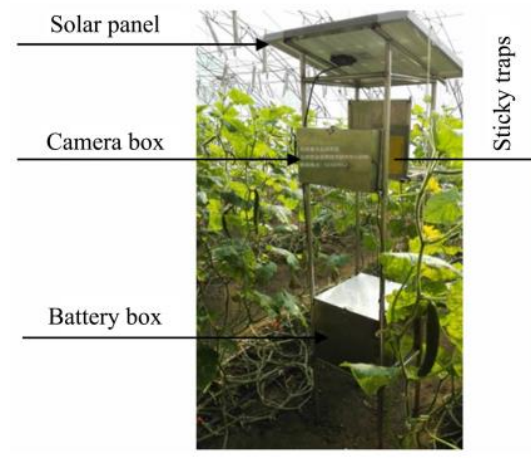

a. Automatic image acquisition system

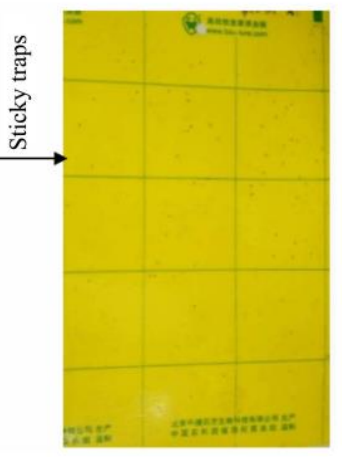

b. Sticky trap

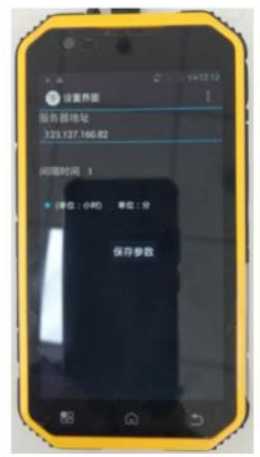

c. T500 industrial mobile phone

Figure 1 The automatic image acquisition device

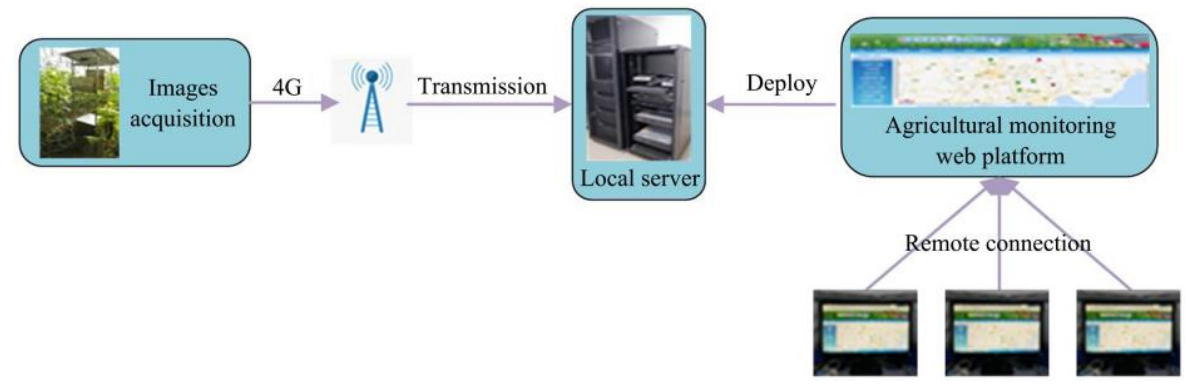

Figure 2 Schematic diagram of the entire system 


\subsection{Image segmentation and recognition algorithms}

\subsubsection{Image segmentation algorithm}

The study proposed a pest image segmentation and counting method in hue-saturation-intensity (HSI) color space and Lab color space to handle the problems caused by non-uniform illumination. The transformation formulas from RGB space to HSI space are as follows $^{[16]}$ :

$$
\begin{gathered}
H=\left\{\begin{array}{l}
\theta, B \leq G \\
360-\theta, B>G
\end{array}\right. \\
S=1-\frac{3}{(R+G+B)}[\min (R, G, B)] \\
I=\frac{1}{3}(R+G+B) \\
\theta=\cos ^{-1}\left\{\begin{array}{l}
0.5[(R-G)+(R-B)] \\
{\left[(R-G)^{2}+(R-G)(G-B)\right]^{\frac{1}{2}}}
\end{array}\right.
\end{gathered}
$$

The RGB color space to Lab color space transformation formulas are as follows:

$$
\left[\begin{array}{l}
X \\
Y \\
Z
\end{array}\right]=\left[\begin{array}{l}
2.76891 .75181 .1302 \\
1.00004 .59070 .0601 \\
0.00000 .56505 .5943
\end{array}\right]\left[\begin{array}{l}
R \\
G \\
B
\end{array}\right]
$$

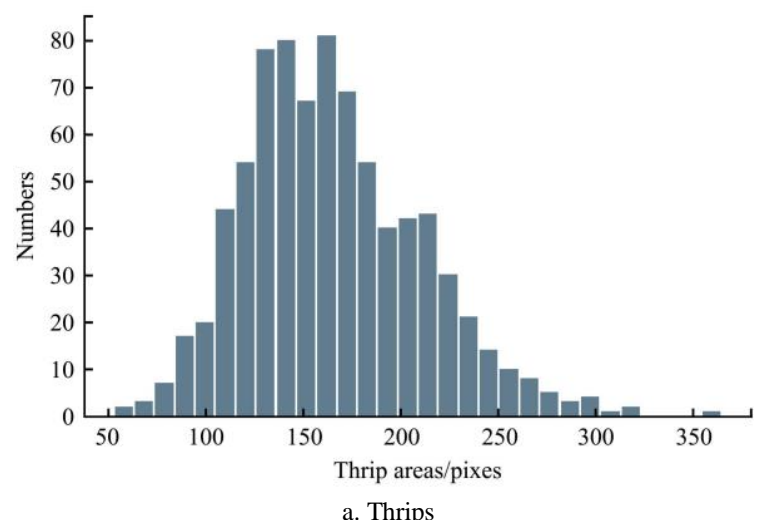

$$
\begin{gathered}
L^{*}=\left\{\begin{array}{l}
116 f\left(\frac{Y}{Y_{0}}\right)^{\frac{1}{3}}-16, \frac{Y}{Y_{0}}>0.008856 \\
903.3 f\left(\frac{Y}{Y_{0}}\right)^{\frac{1}{3}}, \frac{Y}{Y_{0}} \leq 0.008856
\end{array}\right. \\
a^{*}=500\left[f\left(\frac{X}{X_{0}}\right)-f\left(\frac{Y}{Y_{0}}\right)\right] \\
b^{*}=200\left[f\left(\frac{Y}{Y_{0}}\right)-f\left(\frac{Z}{Z_{0}}\right)\right] \\
f(t)=\left\{\begin{array}{l}
\frac{1}{t^{3}}, \quad t>0.008856 \\
7.787 t+\frac{16}{116}, t \leq 0.008856
\end{array}\right.
\end{gathered}
$$

To further improve the pest segmentation accuracy, the study used two steps to preprocess pest images: 1) Target objects were detected by processing the region of interest of the sticky traps based on the distribution of the sampled areas for whiteflies and thrips (Figure 3). Only the target regions between 80 and 1000 pixels were selected.

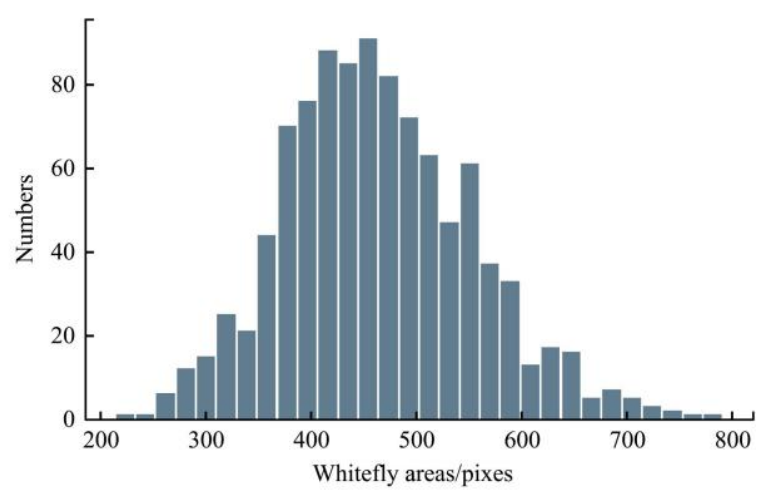

b. Whitefly

Figure 3 Histogram of target pest region sizes

Then, a non-target object area removal algorithm was used to process the pest images (Figure 4). First, the images were converted from RGB to the HSI and Lab color spaces. Second, for components I and B of the respective color spaces, the study used a Prewitt operator and a Canny operator for detection, respectively. Component I of the HSI color space is independent of color. Component B of the Lab color space varies over the range from yellow to blue, forming a strong contrast with the yellow background of the trapped image. Thus, these operators facilitate the edge segmentation of the pests. Finally, by a morphological opening on each detected area, the region of pixels corresponding to the selected area was considered as an object of interest. 2) After segmenting target objects in the HSI and Lab color spaces, the segmented results of the two-color spaces were summed. The result of this segmentation is shown in Section 3.1 .

2.2.2 Image recognition algorithm

2.2.2.1 Feature extraction

The extracted color features (first and second moments) and shape features in the HSI, Lab, and RGB color spaces. Then integrating the three-color features and the morphological features as a feature vector and inputting them to the ensemble learning classification. The corresponding formulas are as follows:
The first moment is

$$
u=\frac{1}{N} \sum_{i=1}^{N} H\left(P_{i}\right)
$$

where, $N$ is the total number of pixels in the pest image area; $P$ and $H\left(P_{i}\right)$ represent the value of the $i$ th pixel in area $P$ in the HSI, RGB, or Lab color space.

The second moment is

$$
\sigma=\sum_{i=1}^{N}\left[\frac{1}{N} \sum_{i=1}^{N}\left(H\left(P_{i}\right)-u\right)^{2}\right]^{\frac{1}{2}}
$$

The second moment signifies the standard variance of the image.

The rectangularity shape feature is

$$
R E=A / S_{M E R}
$$

where, $A=\sum_{i=1}^{n} i$ represents the area of the pest region and $S_{M E R}$ is the minimum enclosing rectangle (MER) of the pest region.

The elongation shape feature is

$$
E l=\frac{l}{w}
$$

where, $l$ is the long axis length and $w$ is the short axis length of the MER of the pest region. 


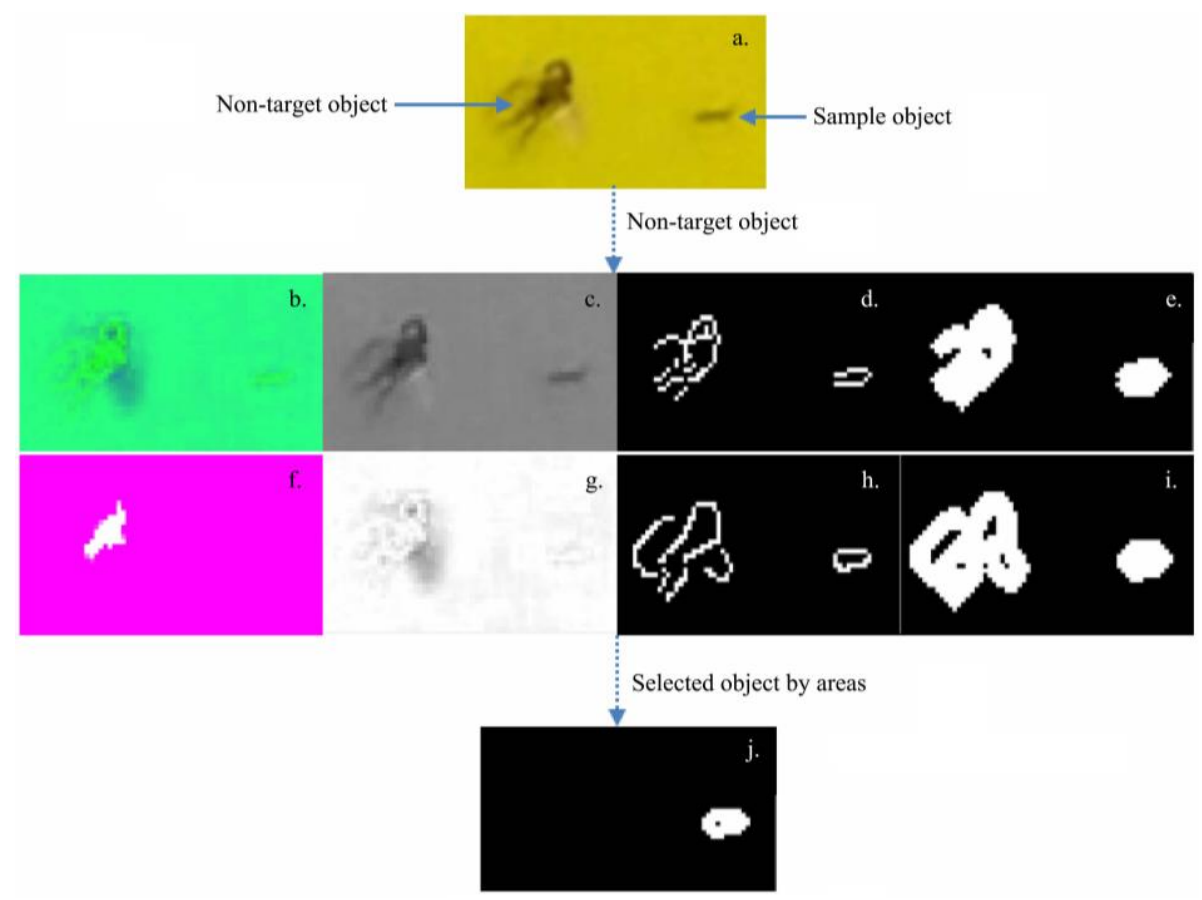

Note: (a) Original image in RGB color space (b) HSI color space image $\quad$ (c) I component (d) Prewitt edge detection (e) Morphological opening

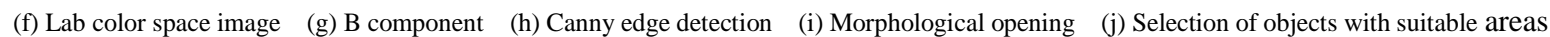

Figure 4 Removal of non-target objects

\subsubsection{Ensemble learning classification}

The ensemble learning classification algorithm is a metaestimator that fits a number of decision tree classifiers on various sub-samples of the dataset. The algorithm steps are as follows:

(1) Assume that the total number of samples is $N$. Then, $n$ $(n<N)$ samples are randomly selected from the $\mathrm{N}$ samples and used to train a decision tree;

(2) Each sample has $M$ attributes. When the nodes of each decision tree need to split, $m(m<M)$ of the most discriminating attributes according to the Gini index are randomly selected as the splitting attributes of the current node;

(3) Each node of each decision tree is split according to the results of step (2) to minimize the impurity of each node until the node cannot be further split;

(4) The results $\left(x_{i}\right)$ are determined by the vote of each tree classifier, that is, the classification formula is as follows:

$$
f\left(x_{i}\right)=\text { majorityvote }\left\{h_{i}\left(x_{i}\right)\right\}_{i=1}^{N t}
$$

The majority vote function implements the majority voting operation and $N t$ denotes the number of decision trees. Figure A1 shows a visual decision tree architecture selected randomly from the trained ensemble learning classifier. The study used $N t=20$ decision trees for the ensemble learning classifier, which usually performs better than a single decision tree. Experiments showed that the ensemble learning classifier obtains state-of-the-art performance on the pest images by comparing its results to those of other classifiers. Finally, the algorithm was integrated into agricultural monitoring networking systems for actual agricultural pest control tasks.

\section{Results and discussion}

\subsection{Pest image segmentation and counting analysis}

The pest images were captured in a real greenhouse environment, and inevitably contain some non-pest material, affecting the counting accuracy. Moreover, the pest images include small objects as well as the gridlines and text on the sticky trap, further complicating the pest object segmentation. Existing object segmentation methods fail to adequately extract the pest objects. In particular, the study showed the results of two common segmentation algorithms: the watershed segmentation algorithm (Figure 5b) and the graph cut algorithm (Figure 5c). Although the watershed segmentation algorithm can remove the gridlines and text on the sticky trap, the object extraction accuracy is low. In contrast, the graph cut algorithm can correctly extract the pest objects but has difficulty removing the gridlines and text. The proposed algorithm achieved superior segmentation and counting results for the pests on the sticky trap, as shown in Figure 5d. Figure 6 shows the counting accuracy for 25 pest images. The study further quantifies the counting accuracy using the following equation:

$$
A E A=\frac{\sum_{i=1}^{N} \frac{N_{i}^{\text {correct }}}{N_{i}^{\text {total }}}}{N}
$$

where, AEA represents the average extraction accuracy of the algorithm with respect to the pest count; $N$ represents the number of pest images $(N=25) ; N_{i}^{\text {total }}$ represents the actual total number of pests in image $i ; N_{i}^{\text {correct }}$ represents the number of target objects extracted by the algorithm. Using Equation (15) over the 25 pest images, the algorithm obtains an accuracy of $91.57 \%$. The false positive extractions are mainly caused by the presence of granular objects of the same size as the pest areas in the image. This problem will be addressed in future research.

The study conducted the pest recognition experiments in three main steps: segmentation of individual pests from the sticky trap images, feature extraction, and the design of the supervised learning model (see Section 2). It is crucial to select an appropriate classification model to maximize the final classification accuracy. Figure 7 shows the confusion matrices corresponding to the three-fold cross-validation results of several supervised classification models. Based on these results, an ensemble learning classification model was selected as our pest recognition model in this study. The confusion matrix presents the number of 
true positive, false positive (in which a pest is detected where it does not exist), true negative, and false negative (in which a pest exists but is not detected) pest detections ${ }^{[29]}$. The diagonal elements of the confusion matrix indicate the true positives and the

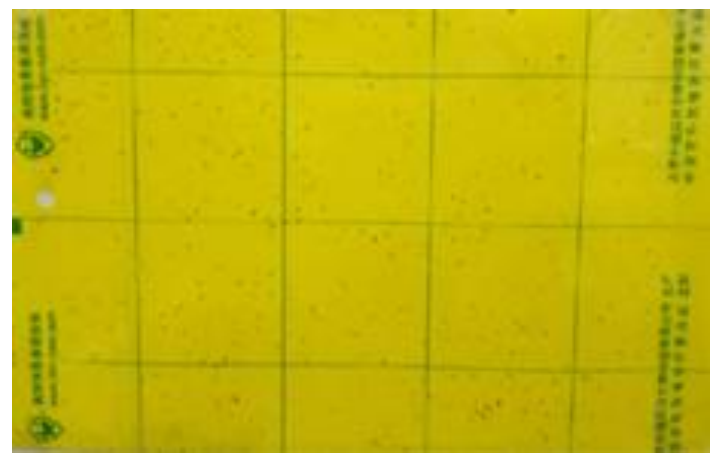

a. Original image

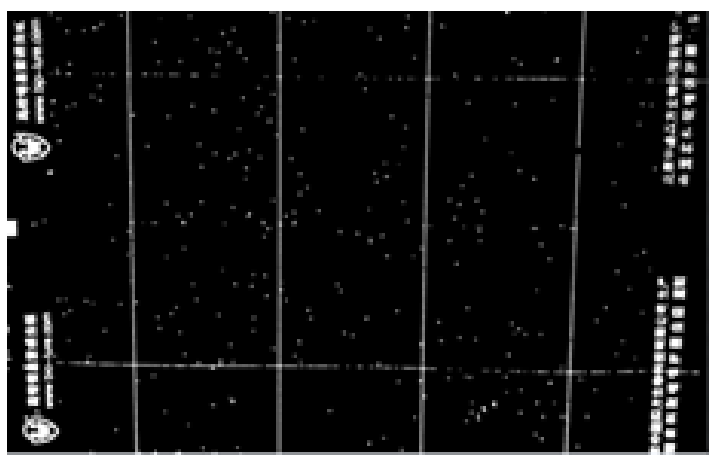

c. Graph cut algorithm rest of the elements in the rows represent the false positives. All of the classifiers show reasonably high true positive and true negative results as well as reasonably low false positive and false negative results.

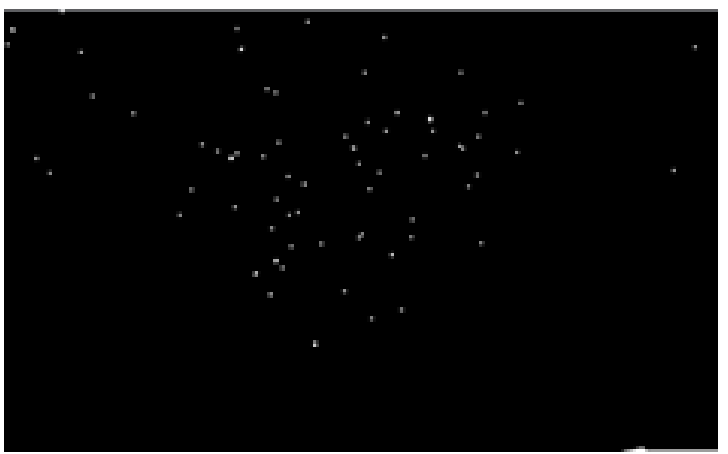

b. Watershed segmentation algorithm

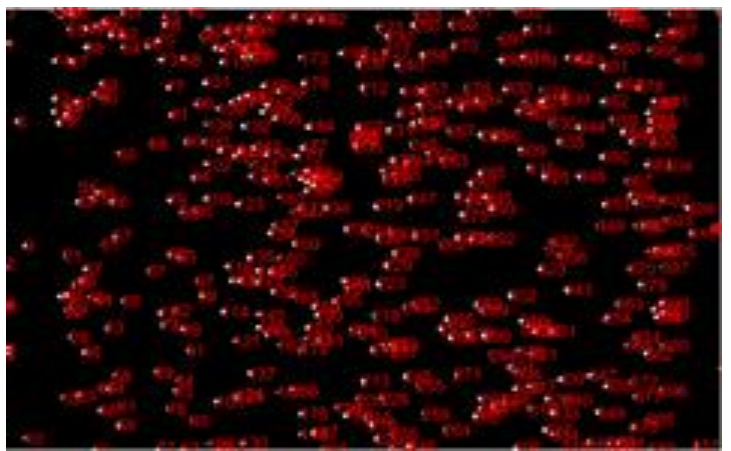

d. Proposed segmentation and counting algorithm

Figure 5 Comparison of image segmentation algorithms

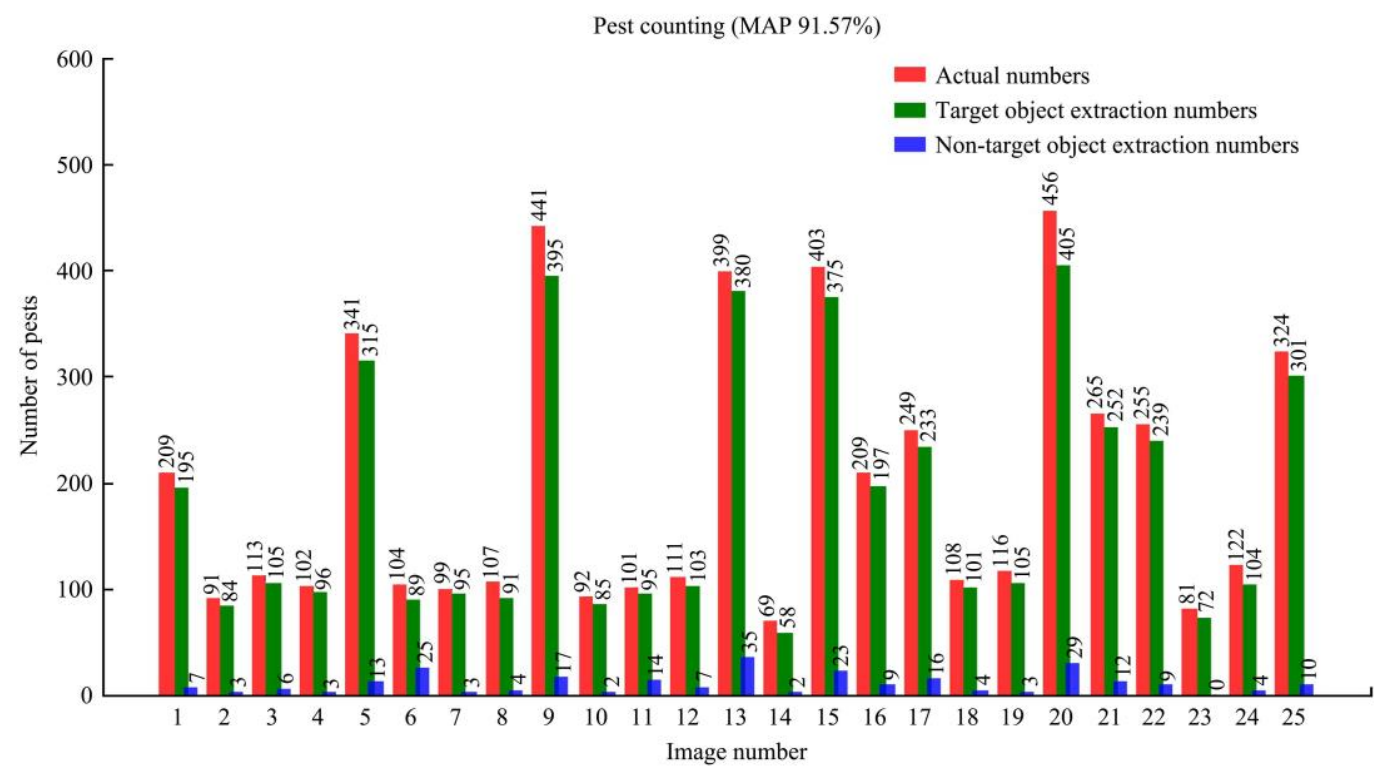

Figure 6 Statistics of pest counting results

The experiment employed accuracy and recall metrics to evaluate the classifier recognition rate ${ }^{[30]}$, and used two threshold-dependent measures: $F_{a}$ score and accuracy:

$$
\begin{gathered}
\text { Precision }=\frac{\text { True positive }}{\text { True positive }+ \text { False positive }} \\
\text { Recall }=\frac{\text { True positive }}{\text { True positive }+ \text { False negative }} \\
F_{\alpha}=\left(1+\alpha^{2}\right) \frac{\text { Precision } \times \text { Recall }}{\alpha^{2} \text { Precision }+ \text { Recall }}
\end{gathered}
$$

Accuracy $=$

True positive + True negative

$\overline{\text { True positive }+ \text { False negative }+ \text { True negative }+ \text { False positive }}$

\subsection{Evaluation of pest classification results}

A precision vs. recall plot shows the trade-off between increasing the number of detected pests (true positives) and reducing the number of false positives. $F_{a}$ score, which is based on precision and recall, represents the overall performance of the pest recognition algorithm, including the false positives and false 
negatives. A larger $F_{a}$ score indicates better performance. The experiment sets $a=1$ for all of the reported results in this study. From the confusion matrices (true positive: true value $=1$, predicted value $=1$; true negative: true value $=0$, predicted value $=0$; false positive: true value $=0$, predicted value $=1$; false negative: true value $=1$, predicted value $=0$ ), finally the ensemble learning classifier achieves the highest $F_{a}$ score and accuracy. Table 1
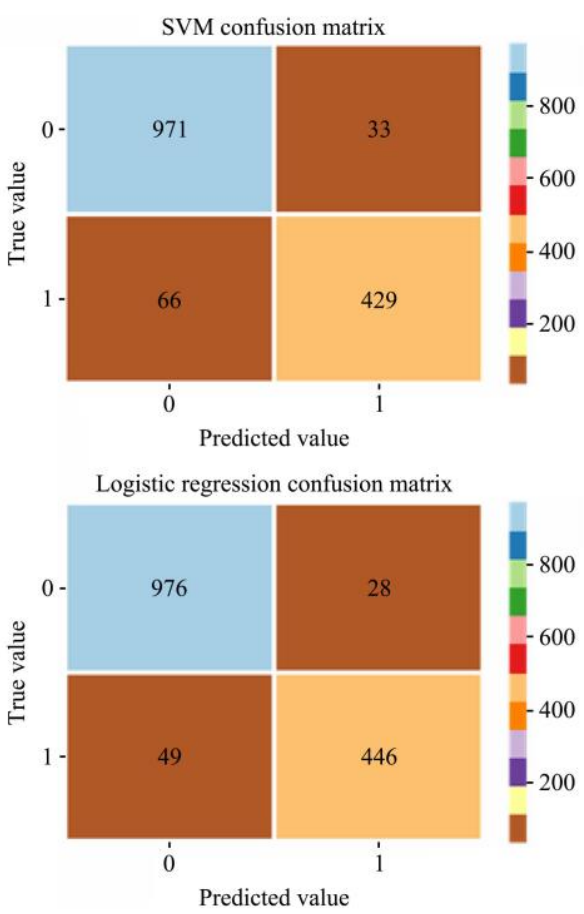

Figure 7 Confusion matrix evaluation: accuracy of pest detection using various classification algorithms

Table 1 Evaluation results of different classification models

\begin{tabular}{lcc}
\multicolumn{1}{c}{ Classifiers } & $F_{1}$ score/\% & Accuracy/\% \\
\hline SVM (RBF kernel,gamma=0.001) & 89.66 & 93.40 \\
$\begin{array}{l}\text { Decision tree (criterion="gini", splitter="best") } \\
\text { Logistic regression (multi_class='multinomial', } \\
\text { solver='lbfgs') }\end{array}$ & 90.67 & 93.86 \\
$\begin{array}{l}\text { Ensemble classification (n_estimators=20, } \\
\text { max_features = 'sqrt') }\end{array}$ & 93.06 & 94.86 \\
\hline
\end{tabular}

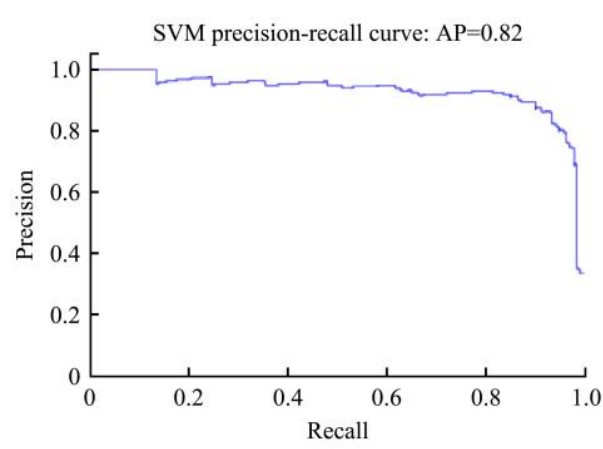

Logistic regression precision-recall curve: $\mathrm{AP}=0.86$

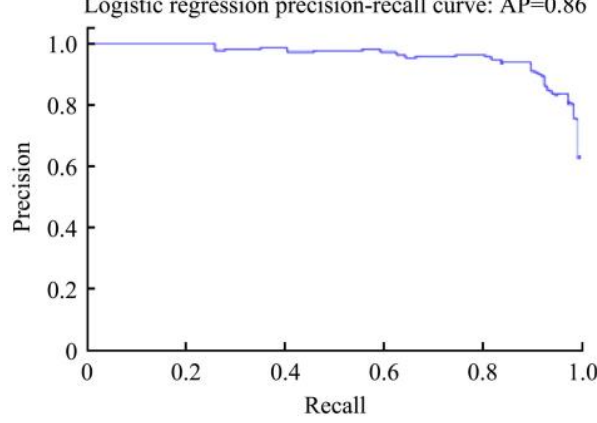

shows the ensemble learning classification algorithm achieves the best classification performance compared with the other algorithms A high accuracy value indicates that the results contain a high percentage of correctly identified pests and a low misclassification percentage. Misclassification occurs frequently overall and affects the recognition results; thus, the accuracy analysis measures the overall reliability of the classification models.
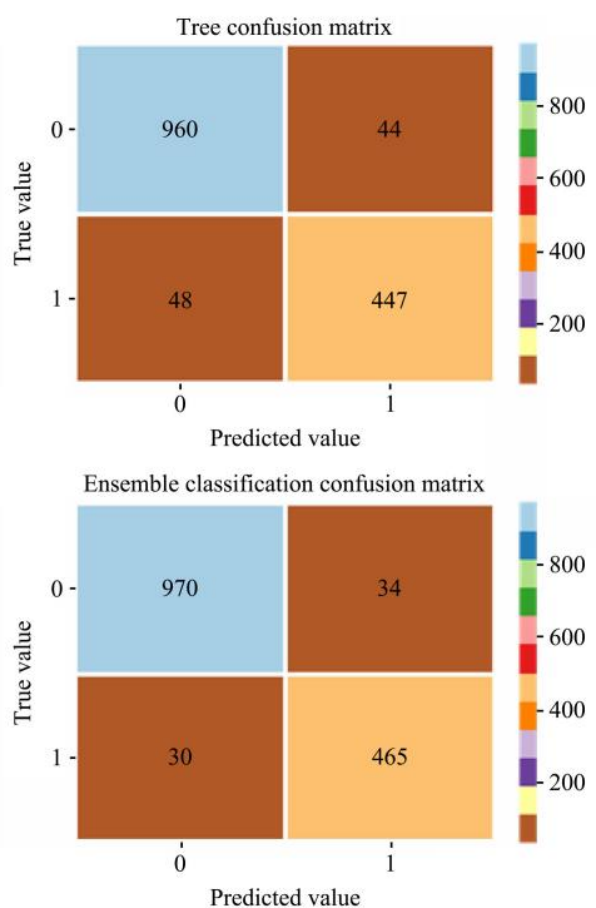

\subsection{Recognition results and analysis of test images}

The experiment randomly selected one sticky trap image and tested all of the above classifiers on this pest image. Figure 8 shows that ensemble learning classification achieves the best precision-recall results. A precision-recall curve typically shows decreasing precision as the recall increases. The ensemble learning classifier achieves superior AP (average precision) results concerning the other classifiers.
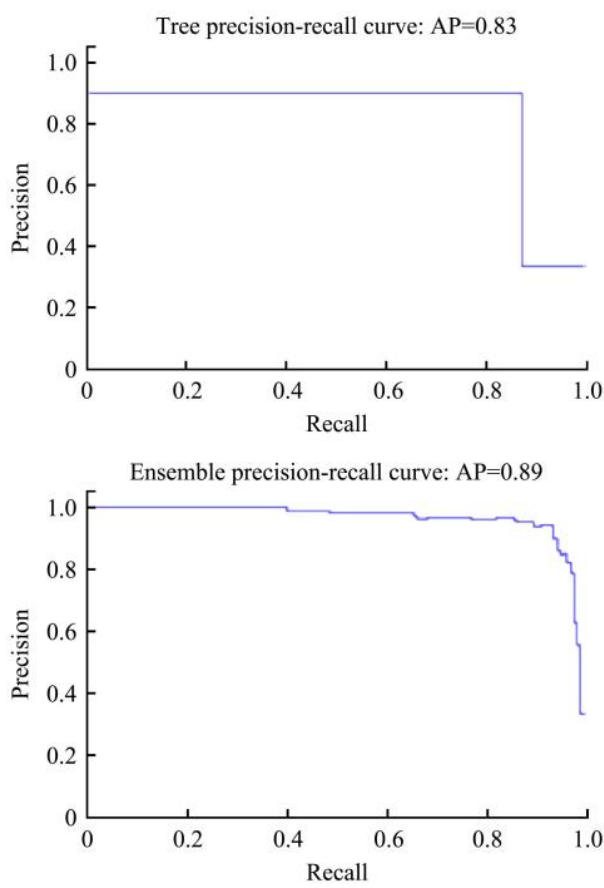

Figure 8 Precision-recall curves of different classifiers 
Figure 9 shows the final recognition and counting results. The labels "1" and " 2 " represent whiteflies and thrips, respectively. The backend server is a single Intel Xeon machine with two quad-core processors running at $2.33 \mathrm{GHz}$ each and $16 \mathrm{~GB}$ of RAM. All of the operations are handled by the server.
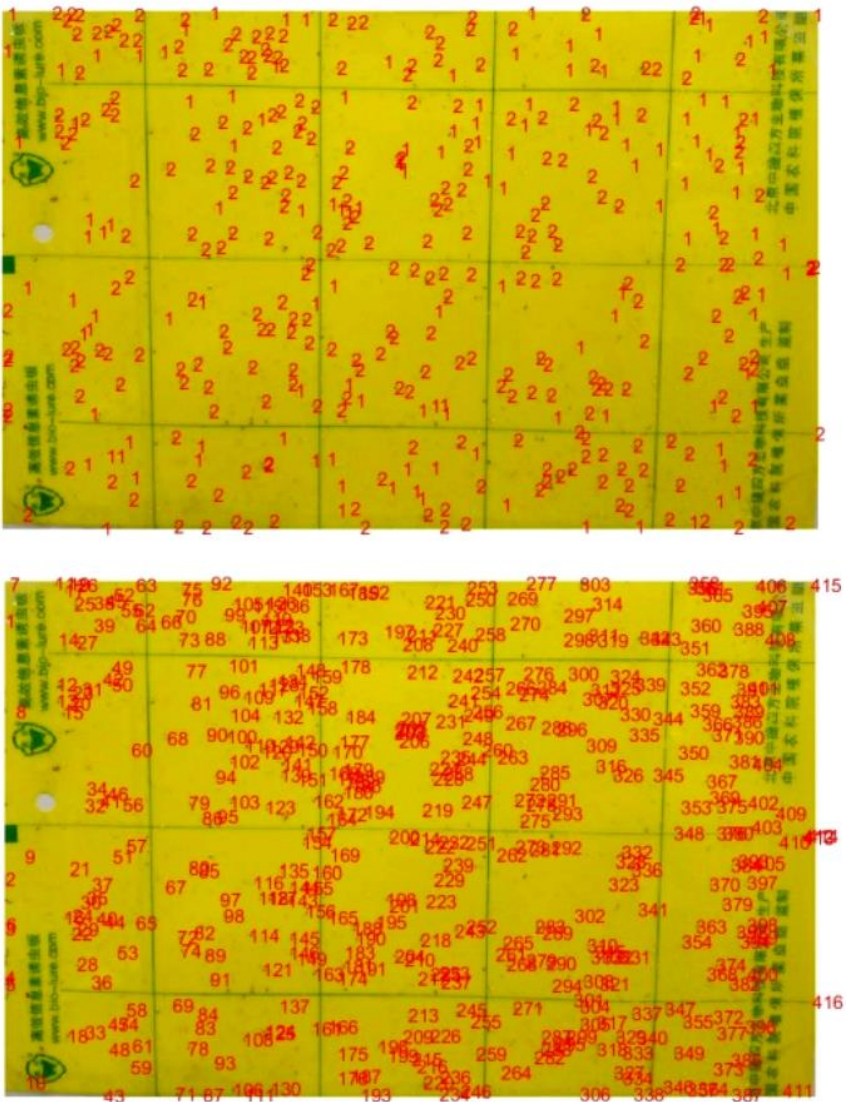

Figure 9 Results of pest recognition and counting

\section{Conclusions}

The greenhouse environment presents particular challenges to automated recognition and counting. These challenges include the low resolution of the images combined with the small size of the target pests and the presence of other materials and markings in the image. This study proposed an algorithm to accurately identify and count pests in images of a sticky trap from a real greenhouse environment. The algorithm achieved state-of-the-art performance. Moreover, a complete end-to-end system was implemented to automate the entire process with little manual intervention for image acquisition, transmission, storage, recognition, and counting.

The study conducted the pest segmentation in the HSI and Lab color spaces and summed the results to further improve the segmentation accuracy. The segmentation on the HSI and Lab color spaces achieved better results than some classical segmentation algorithms. It appeared that in the context of the study, the gridlines and text on the sticky traps showed much lower intensity values in component I of HSI and component B of Lab, thus facilitating the extraction of the pest objects. The color and shape features of the pests in different color spaces were extracted and an ensemble decision trees algorithm was used to perform the recognition task. Experimental results indicated that the method was proposed accurately recognized and counted the small-sized pests in sticky trap images. The complete system including the hardware setup and software design will be of interest to the agriculture industry. In the future, the system will be further improved to false positive detection, and expand the scope of its applicability to fields outside of the greenhouse environment. The difficult problem will also be tried to be solved that locating small pest objects and realize automatic detection for all small pests in sticky traps using a deep learning algorithm.

\section{Acknowledgements}

This work was financially supported by the National Natural Science Foundation of China (Grant No. 61601034) and the National Natural Science Foundation of China (Grant No. 31871525). The authors acknowledge Kimberly Moravec, PhD, from Liwen Bianji, Edanz Editing China (www.liwenbianji.cn/ac), for editing the English text of a draft of this manuscript.

\section{[References]}

[1] Qiao M, Lim J, Ji C W, Chung B K, Kim H Y, Uhm K B, et al. Density estimation of Bemisia tabaci (Hemiptera: Aleyrodidae) in a greenhouse using sticky traps in conjunction with an image processing system. J. Asia-Pacific Entomol, 2008; 11(1): 25-29.

[2] Pinto-Zevallos D M, Vänninen I. Yellow sticky traps for decision-making in whitefly management: what has been achieved? Crop Prot, 2013; 47: 74-84.

[3] Allen W A, Rajotte E G. The changing role of extension entomology in the IPM era. Annu. Rev. Entomol, 1990; 35: 379-397.

[4] Hu Y Q, Song L T, Zhang J, Xie C J, Li R. Pest image recognition of multi-feature fusion based on sparse representation. Pattern Recognition and Artificial Intelligence, 2014; 27(5): 985-992. (in Chinese)

[5] Kang S H, Song S H, Lee S H. Identification of butterfly species with a single neural network system. J. Asia-Pacific Entomol, 2012; 15(3): 431-435.

[6] Kang S H, Cho J H, Lee S H. Identification of butterfly based on their shapes when viewed from different angles using an artificial neural network. J. Asia-Pacific Entomol, 2014; 17(2): 143-149.

[7] Tofilski A. DrawWing, a program for numerical description of insect wings. Journal of Insect Science, 2004; 4(1): 17. doi: 10.1673/ 031.004.1701.

[8] Wang J, Lin C, Ji L, Liang A. A new automatic identification system of insect images at the order level. Knowl.-Based Syst, 2012; 33: 102-110.

[9] Li W Y, Li M, Qian J P, Sun C H, Du S F, Chen M X. Segmentation method for touching pest images based on shape factor and separation points location. Transactions of the Chinese Society of Agricultural Engineering, 2015; 31(6): 175-180. (in Chinese)

[10] Larios N, Soran B, Shapiro L G, Martínez-Muñoz G, Lin J, Dietterich T G. Haar random forest features and SVM spatial matching kernel for stonefly species identification. In: 2010 20th International Conference on Pattern Recognition. Istanbul: IEEE, 2010; pp.2624 - 2627.

[11] Larios N, Deng H L, Zhang W, Sarpola M, Yuen J, Paasch R, et al Automated insect identification through concatenated histograms of local appearance features: feature vector generation and region detection for deformable objects. Machine Vision and Applications, 2008; 19: 105-123.

[12] Martinez-Munoz G, Larios N, Mortensen E, Zhang W, Yamamuro A, Paasch R, et al. Dictionary-free categorization of very similar objects via stacked evidence trees. In: 2009 IEEE Conference on Computer Vision and Pattern Recognition. Miami, FL: IEEE, 2009; pp.549-556.

[13] Lytle D A, Martínez-Muñoz G, Zhang W, Larios N, Shapiro L, Paasch R, et al. Automated processing and identification of benthic invertebrate samples. J. North Am. Benthol. Soc, 2010; 29(3): 867-874.

[14] Boissard P, Martin V, Moisan S. A cognitive vision approach to early pest detection in greenhouse crops. Comput. Electron. Agric, 2008; 62(2): 81-93.

[15] Xia C L, Chon T S, Ren Z M, Lee J M. Automatic identification and counting of small size pests in greenhouse conditions with low computational cost. Ecol Inform, 2015; 29(6): 139-146.

[16] Wang X, Hänsch R, Ma L Z, Hellwich O. Comparison of different color spaces for image segmentation using graph-cut. In: 2014 International Conference on Computer Vision Theory and Applications. Lisbon: IEEE, 2014; pp.301-308. 
[17] Maharlooei M, Sivarajan S, Bajwa S G, Harmon J P, Nowatzki J. Detection of soybean aphids in a greenhouse using an image processing technique. Comput. Electron. Agric, 2017; 132: 63-70.

[18] Ebrahimi M A, Khoshtaghaza M H, Minaei S, Jamshidi B. Vision-based pest detection based on SVM classification method. Comput. Electron. Agric, 2017; 137(6): 52-58.

[19] Sun Y R, Cheng H, Cheng Q, Zhou H Y, Li M H, Fan Y H, et al. A smart-vision algorithm for counting whiteflies and thrips on sticky traps using two-dimensional Fourier transform spectrum. Biosystems Engineering, 2017; 153: 82-88.

[20] Heinz K M, Parrella M P, Newman J P. Time-efficient use of yellow sticky traps in monitoring insect populations. Journal of Economic Entomology, 1992; 85(6): 2263-2269.

[21] Steiner M Y, Spohr L J, Barchia I, Goodwin S. Rapid estimation of numbers of whiteflies (Hemiptera: Aleurodidae) and thrips (Thysanoptera: Thripidae) on sticky traps. Australian Journal of Entomology, 1999; 38: 367-372.

[22] Cho J, Choi J, Qiao M, Ji C W, Kim H Y, Uhm K, et al. Automatic identification of whiteflies, aphids and thrips in greenhouse based on image analysis. International Journal of Mathematics and Computers in Simulation, 2007; 1(1): 46-53.

[23] Espinoza E, Valera D L, Torres J A, López A, Molina-Aiz F D. Combination of image processing and artificial neural networks as a novel approach for the identification of Bemisia tabaci and Frankliniella occidentalis on sticky traps in greenhouse agriculture. Comput. Electron. Agric, 2016; 127(3): 495-505

[24] Solis-Sánchez L O, García-Escalante J J, Castañeda-Miranda R, Torres-Pacheco I, Guevara-González R. Machine vision algorithm for whiteflies (Bemisia tabaci Genn.) scouting under greenhouse environment. Journal of Applied Entomology, 2009; 133: 546-552.

[25] Solis-Sánchez L O, Castañeda-Miranda R, García-Escalante J J, Torres-Pacheco I, Guevara-González R G, Castañeda Miranda C L, et al. Scale invariant feature approach for insect monitoring. Computers and Electronics in Agriculture, 2011; 75(1): 92-99.

[26] Lowe D G. Distinctive image features from scale-invariant keypoints International Journal of Computer Vision, 2004; 60: 91-110.

[27] Xiao D Q, Feng J Z, Lin T Y, Pang C H, Ye Y W. Classification and recognition scheme for vegetable pests based on the BOF-SVM model. Int J Agric \& Biol Eng, 2018; 11(3): 190-196.

[28] Deng L M, Wang Y J, Han Z Z, Yu R S. Research on insect pest image detection and recognition based on bio-inspired methods. Biosystems Engineering, 2018; 169(8): 139-148.

[29] Kohavi R, Provost F. Glossary of terms: special issue on applications of machine learning and the knowledge discovery process. Mach. Learn, 1998; 30: 271-274.

[30] Xia C L, Lee J M, Li Y, Chung B K, Chon T S. In situ detection of small-size insect pests sampled on traps using multifractal analysis. Optical Engineering, 2012; 51(2): 027001. doi: 10.1117/1.OE.51.2.027001.

\section{Appendix}

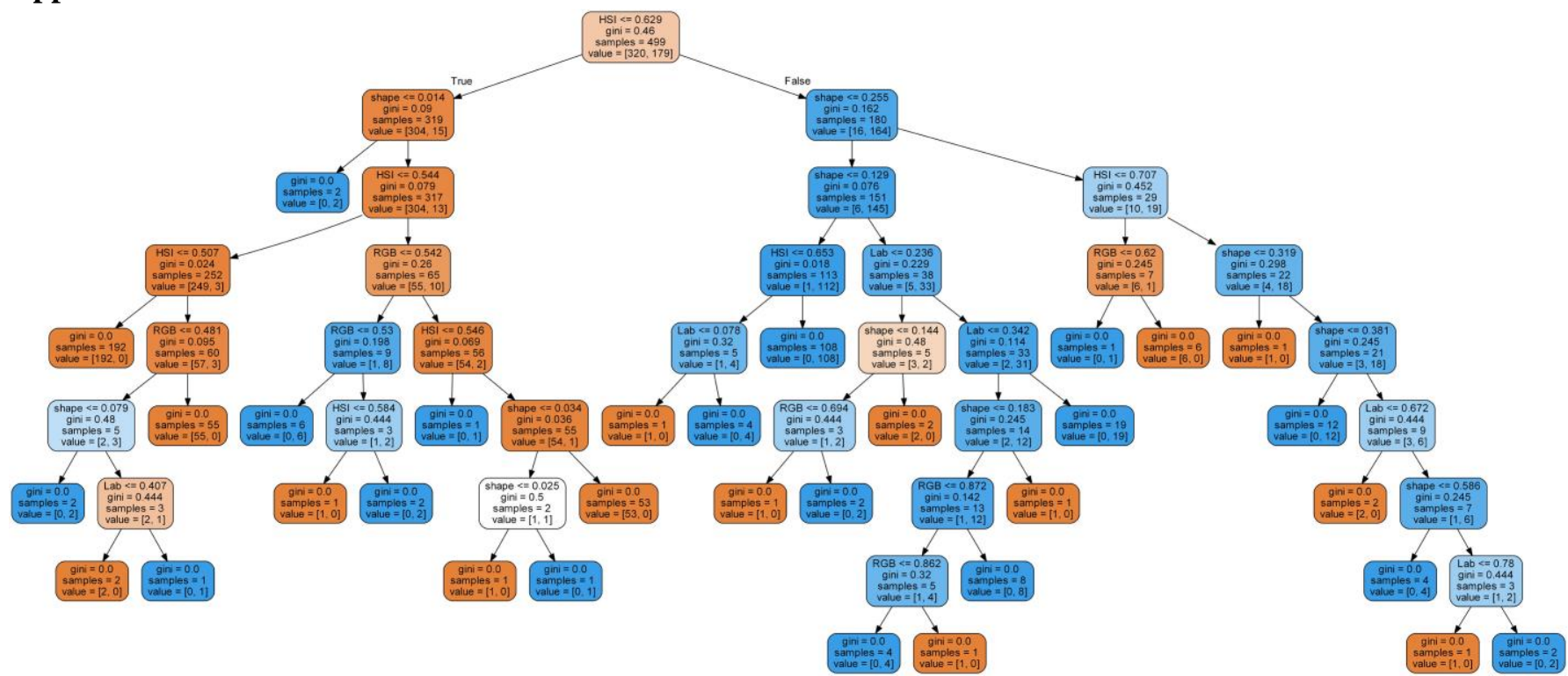

Note: Each node calculates its optimal splitting criterion according to the color and shape features we extracted. As the nodes split further, the sample class impurity (Gini) gradually decreases. Finally, the samples are classified by the value shown in the leaf nod

Figure A1 Visualization of a decision tree randomly selected from the trained ensemble learning classifier 